Supporting Information

\title{
Human cytomegalovirus infection activates glioma activating transcription factor 5 via microRNA in stress-induced manner
}

Ming $\mathrm{Hu}^{1}$, Bo $\mathrm{Yu}^{1}$, Bingxu Zhang ${ }^{1}$, Bin Wang ${ }^{1}$, Dongmeng Qian ${ }^{1}$, Huanting $\mathrm{Li}^{2}$, Junwei $\mathrm{Ma}^{2 *}$, and David $\mathrm{X} \mathrm{Liu}{ }^{3 *}$

1 Department of Special Medicine, Basic Medicine College, Qingdao University, Qingdao, Shandong 266071, P. R.China

2 Department of Neurosurgery, The Affiliated Hospital of Qingdao University, Qingdao University, Qingdao, Shandong 266000, P. R.China

3 Department of Natural Sciences, College of Science and Health Professions, Northeastern State University, Broken Arrow, OK 74014, USA

Co-corresponding authors: Junwei Ma (qdmajw@outlook.com), Department of Neurosurgery, the Affiliated Hospital of Qingdao University Qingdao, Shandong, P. R.China. and David X Liu (liud@nsuok.edu), Department of Natural Sciences, College of Science and Health Professions, Northeastern State University, Broken Arrow, OK 74014, USA

Table S1 Upregulated genes enriched in GO terms

ID Description qvalue

GO:007030 positive regulation of stress-activated protein kinase signaling 0.00020

4 cascade

GO:003287

0.00038

positive regulation of stress-activated MAPK cascade 4 
GO:006219

cellular response to chemical stress

7

GO:007122

cellular response to acid chemical

9

GO:005073

positive regulation of peptidyl-tyrosine phosphorylation

GO:190533

positive regulation of morphogenesis of an epithelium

2

GO:003459

cellular response to oxidative stress

9

GO:007177

cellular response to BMP stimulus

3

GO:007121

cellular response to biotic stimulus

6

GO:000961

response to mechanical stimulus

2

GO:007133

cellular response to glucose stimulus

3

GO:003241

positive regulation of transporter activity

1

GO:009723

cellular response to toxic substance

0.00256

0.00072

1

0.00073

1

0.00099

8

0.00155

8

0.00162

2

0.00193

1

7

0.00387

5

0.00748

8

0.00816

4

0.00820

6 
GO:003166

cellular response to extracellular stimulus

8

GO:003413

toll-like receptor 2 signaling pathway

4

GO:000288

regulation of myeloid leukocyte mediated immunity

6

GO:005098

detection of mechanical stimulus

2

GO:190012

regulation of receptor binding

0

GO:200037

positive regulation of reactive oxygen species metabolic process

9

GO:003649

PERK-mediated unfolded protein response

9

GO:004578

positive regulation of cell adhesion

5

GO:003227

regulation of protein polymerization

1

GO:000181

positive regulation of cytokine production

9

GO:004254

response to hydrogen peroxide
0.01021

0.01876

2

0.01939

0.01982

4

0.02070

7

0.0226

0.02314

4

0.02415

6

0.02559

7

0.03007

3

0.03095 2 
GO:007030

cellular response to hydrogen peroxide

1

GO:003462

cellular response to unfolded protein

0

GO:006220

regulation of pattern recognition receptor signaling pathway

7

GO:005109

positive regulation of DNA-binding transcription factor activity

1

GO:000167

cellular glucose homeostasis
0.03248

8

0.03545

6

0.03704

9

0.03764

5

0.00937 
Table S2 Downregulated genes enriched in GO terms

\begin{tabular}{|c|c|c|}
\hline ID & Description & qvalue \\
\hline \multirow[t]{2}{*}{ GO:005080 } & & 0.00000 \\
\hline & synapse organization & \\
\hline 8 & & 0 \\
\hline \multirow[t]{2}{*}{ GO:000740 } & & 0.00000 \\
\hline & axonogenesis & \\
\hline 9 & & 0 \\
\hline GO:009874 & cell-cell adhesion via plasma-membrane adhesion & 0.00000 \\
\hline 2 & molecules & 0 \\
\hline \multirow[t]{2}{*}{ GO:009917 } & & 0.00000 \\
\hline & regulation of trans-synaptic signaling & \\
\hline 7 & & 0 \\
\hline \multirow[t]{2}{*}{ GO:005080 } & & 0.00000 \\
\hline & regulation of synapse structure or activity & \\
\hline 3 & & 0 \\
\hline \multirow[t]{2}{*}{ GO:000741 } & & 0.00000 \\
\hline & synapse assembly & \\
\hline 6 & & 0 \\
\hline \multirow[t]{2}{*}{ GO:005080 } & & 0.00000 \\
\hline & regulation of synapse organization & \\
\hline 7 & & 0 \\
\hline \multirow[t]{2}{*}{ GO:004232 } & & 0.00013 \\
\hline & negative regulation of phosphorylation & \\
\hline 6 & & 3 \\
\hline \multirow[t]{2}{*}{ GO:004592 } & & 0.00018 \\
\hline & negative regulation of growth & \\
\hline 6 & & 4 \\
\hline \multirow[t]{2}{*}{ GO:004804 } & & 0.00018 \\
\hline & focal adhesion assembly & \\
\hline 1 & & 5 \\
\hline
\end{tabular}


GO:007037

ERK1 and ERK2 cascade

1

GO:000715

neuron cell-cell adhesion

8

GO:007099

neuron death

7

GO:190121

regulation of neuron death

4

GO:004544

endothelial cell differentiation

6

GO:004507

negative regulation of viral genome replication

1

GO:004230

negative regulation of protein import into nucleus

8

GO:190459

negative regulation of protein import

0

GO:002240

regulation of cell-cell adhesion

7

GO:001714

stem cell division
0.00028

6

0.00047

7

0.00222

0

0.00840

9

0.01098

1

0.01452

4

0.01651

2

0.01651

2

0.01907

7

0.02101

1 